\title{
Quelle contre-révolution en Tunisie et en Egypte ? L'héritage du RCD et du PND dans les systèmes partisans et le secteur de l'économie informelle ${ }^{1}$
}

\author{
Clément Steuer et Adrien Doron. CNRS (Ladyss/UMR 7533)/ERC TARICA
}

Le Rassemblement constitutionnel démocratique (RCD) en Tunisie, et le Parti national démocratique (PND) en Égypte, ont dominé la vie politique de leurs pays respectifs durant plusieurs décennies. À la veille de la révolution, ces deux partis constituaient à la fois l'espace dans lequel s'affrontaient les projets de société concurrents portés par les élites, et l'un des principaux liens entre le régime et la société. La crise de ces deux fonctions (réformatrice et clientéliste) explique l'inaptitude de ces deux formations à survivre dans le contexte d'ouverture politique consécutif à la chute des dictateurs. Dans cette perspective, leur dissolution par la justice a probablement accéléré leur dislocation plutôt qu'elle ne l'a provoquée. En effet, la lutte intestine opposant - au sein de chacune de ces deux formations - conservateurs et réformateurs s'est traduite dans ce nouveau contexte par la mise en concurrence sur le marché électoral de différents projets de restauration autoritaire ou de réformes graduelles du régime. Par ailleurs, l'absence de ligne idéologique claire manifestée par ces deux partis a favorisé la dispersion d'une partie de leur clientèle et leur réalignement sur des lignes de clivage fonctionnelles ou culturelles. Les réseaux informels liés aux anciens partis ont néanmoins su survivre dans le champ économique, au prix d'une recomposition.

The Constitutional Democratic Rally (RCD) in Tunisia, and the National Democratic Party (NDP) in Egypt, had dominated the political life of their respective countries for decades. Prior to the revolution, they constituted at the same time a space of competition for the different elites-backed projects, and one of the main linkages between the state and the society. The crisis of both functions (reformist and clientelist) explains the failure of both organizations to survive in a context of political opening following the overthrowing of the dictators. In this respect, their judicial disbanding had been an accelerator of their disintegration rather than a cause. Indeed, the internal struggle opposing - in both parties - conservatives and reformers has resulted in the formulation of different projects (authoritarian restoration or step-by-step reform of the old regimes) competing on the electoral market. In addition, the lack of a clear ideological identity for both organizations has favored the dispersion of part of their former members, and their realignment on functional or cultural cleavages. Nevertheless, the informal networks linked to the former parties have survived in the economical field, by means of a reshaping.

Si les trajectoires révolutionnaires de la Tunisie et de l’Égypte ont divergé de manière spectaculaire à partir de 2013, il n’en reste pas moins que leurs partis hégémoniques respectifs - le Rassemblement constitutionnel démocratique (RCD) en Tunisie, et le Parti national démocratique (PND) en Égypte, - n’ont jamais été restaurés, et que les deux pays disposent, en 2020, d'un système partisan fragmenté. En outre, dans les deux pays, toutes les tentatives d'exclure de la vie politique les membres des ex-partis dirigeants ont échoué. Néanmoins, ces deux organisations n’ont pas été reconstituées pour autant, et leurs anciens cadres, élus et militants se sont dispersés dans une grande partie du champ partisan. En effet, malgré les appels du RCD à " construire une nouvelle démocratie qui n’exclut personne », quelques jours à peine après la chute de Ben $\mathrm{Ali}^{2}$, ses activités furent suspendues dès le 6 février 2011. Le 9 mars, le tribunal de premier instance de Tunis décidait de sa dissolution, au motif de mise en danger de la stabilité du pays. De son côté, le PND s'était doté le 12 avril 2011 d'une nouvelle direction, clamant sa volonté de tourner la page de l'ancien régime, d'embrasser les «principes de la révolution » et de prendre sa place dans la nouvelle ère démocratique qui s'annonçait. Le 16 avril, il était pourtant lui aussi dissous par décision de justice, au motif de « corruption de la vie politique ».

Ces deux partis, qui ont dominé la vie politique de leurs pays respectifs durant plus de vingt ans - trente pour le PND -, ont paradoxalement toujours été des objets sous-étudiés. Cette situation peut sans doute en partie

1 Cette publication a bénéficié du soutien du Conseil européen de la recherche, Programme Horizon 2020, projet ERC TARICA convention $\mathrm{n}^{\circ} 695674$.

2 Le Point, 20 janvier 2011. https://www.lepoint.fr/monde/tunisie-dissolution-du-rcd-ex-parti-au-pouvoir-20-01-20111286170 24.php 
s'expliquer par l'absence de ligne politique claire, caractéristique des partis hégémoniques ${ }^{3}$, qui aura marqué ces deux organisations depuis leurs origines. En effet, contrairement au parti unique des régimes totalitaires, destiné à mobiliser la société autour d’un projet politique, le parti hégémonique n'accorde pas une place centrale à l'idéologie, et représente même l'un des principaux outils de dépolitisation de la société aux mains des gouvernants. Là où un parti hégémonique existe, il constitue généralement une pièce maîtresse du dispositif autoritaire, en jouant un double rôle de mise en cohérence du bloc historique et de cooptation des élites - notamment locales - et de leur intégration au sein du régime.

Les trop rares travaux produits sur le RCD et le PND se sont concentrés soit ${ }^{4}$ sur leur potentiel modernisateur et réformateur, ainsi que sur leurs capacités de mobilisation, soit ${ }^{5}$ sur leur rôle dans les systèmes clientélistes qui soutenaient les deux régimes ${ }^{6}$. À la veille de la révolution, ces deux partis constituaient en effet à la fois l'espace dans lequel s'affrontaient les projets de société concurrents portés par les élites, et l'un des principaux liens entre le régime et la société. Notre thèse est que la crise de ces deux fonctions (réformatrice et clientéliste) a non seulement constitué l'une des causes des révolutions de 2011- ou en tout cas l'une des conditions les rendant possibles -, mais explique également l'inaptitude de ces deux formations à survivre dans le contexte d'ouverture politique consécutif à la chute des dictateurs. Dans cette perspective, leur dissolution par la justice a probablement accéléré leur dislocation plutôt qu'elle ne l'a provoquée. En effet, la lutte intestine opposant - au sein de chacune de ces deux formations - conservateurs et réformateurs s'est traduite dans ce nouveau contexte par la mise en concurrence sur le marché électoral de différents projets de restauration autoritaire ou de réformes graduelles du régime. Par ailleurs, l'absence de ligne idéologique claire manifestée par ces deux partis a favorisé la dispersion d'une partie de leur clientèle et leur réalignement sur des lignes de clivage fonctionnelles ou culturelles ${ }^{7}$.

\section{Des luttes intestines à la compétition électorale}

La Tunisie et l’Égypte ont toutes deux connu des régimes à partis uniques installés au cours des années 1950 à la faveur de la lutte contre l'impérialisme et de la popularité des modèles socialistes de développement : le Parti socialiste destourien (PSD) de Habib Bourguiba, et l'Union socialiste arabe de Gamal Abdel Nasser. Ces deux partis furent transformés en partis hégémoniques par les successeurs de leurs fondateurs respectifs, Anouar al-Sadate et Zine el-Abidine Ben Ali, qui ne se contentèrent pas d'en changer les noms, mais présidèrent également à leur restructuration, et mirent en place les conditions d'un multipartisme limité. Cependant, l’abandon du socialisme et le caractère non-assumé du ralliement au libéralisme vidèrent ces

3 Définis comme des partis dirigeants qui acceptent l'existence de partis minoritaires tout en refusant la possibilité même d'une alternance, n’hésitant pas à recourir à la fraude ou à la violence pour perpétuer leur domination (G. Sartori, Parties and Party Systems. A Framework for Analysis, Cambridge, Cambridge University Press, 1976, p. 230-231).

4 Dans le cas tunisien : V. Geisser et É. Gobe, «Un si long règne... Le régime de Ben Ali vingt ans après », L’Année du Maghreb, n 4, 2008, p. 347-381 ; S. Erdle, Ben Ali's 'New Tunisia'(1987-2009): A Case Study of Authoritarian Modernization in the Arab World, Berlin, Klaus Schwarz, 2010 ; A. Wolf, "'Dégage RCD!' The rise of internal dissent in Ben Ali's Constitutional Democratic Rally and the Tunisian uprisings », Mediterranean Politics, vol. 23, $\mathrm{n}^{\circ}$ 2, p. 245-264, 2018. Pour le cas de l’Égypte : V. Collombier, « The Internal Stakes of the 2005 Elections: The Struggle for Influence in Egypt’s National Democratic Party », Middle East Journal, vol. 61, n 1, 2007, p. 95-111 ; A. Heiss, « The Failed Management of a Dying Regime: Hosni Mubarak, Egypt's National Democratic Party, and the January 25 Revolution », Journal of Third World Studies, vol. 29, n 1, 2012, p. 155-171.

5 Dans le cas tunisien : B. Hibou, La force de l'obéissance. Économie politique de la répression en Tunisie, Paris, La Découverte, 2006 ; M. Ben Romdhane, Tunisie. État, économie et société, Paris, Publi-Sud, 2011 ; A. Allal, « Le 'prix’ de la Révolution en Tunisie », Savoir/Agir, n 34, 2015, p. 117-122. Pour le cas de l’Égypte : M. Kassem, In the Guise of Democracy: Governance in Contemporary Egypt, Reading, Ithaca Press, 1999 ; S. Ben Néfissa et A. A. Arafat, Vote et démocratie dans l'Égypte contemporaine, Paris, Khartala-IRD, 2005 ; T. Aclimandos, "Splendeurs et misères du clientélisme », Égypte/Monde arabe, $3^{\mathrm{e}}$ série, n 7, 2010, p. 197-219 ; I. Farag, «Corrompre, fidéliser : Les ressorts "légitimes”de la compétition électorale égyptienne », Égypte/Monde arabe, $3^{e}$ série, $\mathrm{n}^{\circ}$ 7, 2010, p. 21-46.

6 À noter que certains travaux essaient de lier les deux dimensions : A. A.-D. Arafat, Hosni Mubarak and the Future of Democracy in Egypt, New York : Palgrave MacMillan, 2009 ; L. Blaydes et S. El Tarouty, « La concurrence interne au Parti national démocrate égyptien », Égypte/Monde arabe, $3^{\mathrm{e}}$ série, n 7, 2010, p. 69-93.

7 Sur ces notions, voir S. M. Lipset et S. Rokkan, Structures de clivages, systèmes de partis et alignement des électeurs : une introduction, Bruxelles, Éditions de l'université de Bruxelles, 2008. 
deux partis de leurs prétentions idéologiques ${ }^{8}$. Du fait de leur dépolitisation, et de l'impossibilité d'une véritable alternance, ils devinrent à partir des années 1990 le théâtre de l'affrontement de projets concurrents divisant les élites des deux pays. Suite à la révolution, cette division s'est traduite par l'éclatement de l'héritage des deux formations entre plusieurs partis politiques et candidats à l'élection présidentielle.

Une faction libérale, déçue par les promesses de libéralisation politique portées par Ben Ali au début de son règne (1987-1989) s'était constituée au sein du RCD dès les années 1990. Néanmoins, la crainte de ne pas obtenir l'investiture du parti lors des élections législatives suffisait à la tenir au silence, ses membres se décrivant parfois comme "prisonniers» de leur parti ${ }^{9}$. Au sein du PND, la fin des années 1990 voit apparaître au grand jour un conflit opposant une « ancienne garde » de dirigeants blanchie sous le harnais de l'Union socialiste arabe, à un groupe adverse de quadragénaires proches des milieux d'affaires (dont la figure emblématique était le fils puîné du président, Gamal Moubarak), ainsi que des universitaires désireux de moderniser le pays. Le projet de réforme de cette « jeune garde » consistait en une application des recettes néo-libérales, une l'amélioration du respect des droits formels, et un recours plus fréquent au suffrage universel.

Si Gamal Moubarak et ses proches firent partie des premiers à être arrêtés au cours de la révolution de janvier 2011. Ce sont néanmoins d'autres réformateurs qui s'emparèrent de la direction du parti en avril 2011. Suite à la démission de Hosni Moubarak, jusqu'alors président du PND, l'un des neveux du président Sadate et ancien député indépendant dans l'Assemblée de 2005, Talaat al-Sadate, fut en effet élu pour diriger le parti. Cette élection constitua une surprise, du fait que Talaat al-Sadate n'était jusqu'alors pas membre de cette formation, et était même considéré comme un opposant au régime. Après son élection à la tête du PND, il déclara qu'il allait purger ce dernier de ses éléments corrompus, inclure les idées et principes de la révolution de Janvier dans son programme, et le rebaptiser Nouveau parti national. La dissolution de l'ancien parti hégémonique l'empêcha néanmoins d'appliquer ce programme.

Son frère Esmat al-Sadate avait de son côté cherché à créer un parti réformateur d'opposition en 2009, le parti de la Réforme et du développement (PRD), légalisé en mai 2011. Du fait du soutien qu’il s'empressa d'apporter au Conseil supérieur des forces armées - qui avait pris le pouvoir suite à la chute de Moubarak le PRD fut souvent accusé de constituer un véhicule pour les anciens du PND. De fait, ses prises de position le plaçaient à la confluence des anciens réformateurs les plus critiques du PND et des plus modérés des libéraux. En 2011 toujours, de nombreux petits partis politiques furent créés par d'ancien membres du $\mathrm{PND}^{10}$, tels que le parti national d'Égypte, fondé en août 2011 par Talaat al-Sadate, ou encore le parti de la Liberté et le parti des Conservateurs. C'est en ordre dispersé que ces organisations affrontèrent en 2011 le verdict des urnes. Alors que le PRD parvint à remporter 9 sièges (1,8 \% des élus) avec plus de 600000 électeurs, le parti de la Liberté et le parti national d'Égypte n'obtinrent que 5 sièges chacun. À ce stade, les partis issus du PND apparaissaient ainsi comme faibles, divisés, et peu attrayants pour les électeurs.

En Tunisie, les anciens hauts responsables du régime de Ben Ali furent interdits de se présenter à l'élection de l'Assemblée constituante de 2011, mais ne firent l'objet d'aucune mesure depuis lors ${ }^{11}$. Cette interdiction n'empêcha d'ailleurs pas l'élection de 5 députés de l'Initiative nationale destourienne (Al-Moubadara), parti fondé par Kamel Morjane - ministre de la Défense de Ben Ali de 2005 à 2010 - dans le but de reconvertir dans le système politique post-révolutionnaires d'anciens cadres du RCD ralliés à la démocratie libérale ${ }^{12}$. Après avoir absorbé d'autres petits partis en 2013, l'Initiative obtint encore 3 députés lors des élections de 2014. Il faut dire qu'elle avait alors dû faire face à la concurrence de Nidaa Tounes, parti fondé en 2012 par Beji Caïd Essebsi - ancien ministre de Bourguiba et Premier ministre d'un gouvernement de transition en

8 S. Khiari, Tunisie. Le délitement de la Cité, Paris, Karthala, 2004, p. 75-100 ; J.-N. Ferrié, « Les ressorts de l'équilibre politique durant la présidence de Hosni Moubarak », in V. Battesti et F. Ireton, L'Égypte au présent. Inventaire d'une société avant révolution, Paris, Sindbad/Actes Sud, 2011, p. 323-341, p. 324

9 M. Angrist, « Parties, parliament and political dissent in Tunisia », The Journal of North African Studies, vol. 4, $\mathrm{n}^{\circ}$ 4, 1999, p. 89-104, p. 99.

10 Jadaliyya, 18 novembre 2011, <http://www.jadaliyya.com/pages/index/3163/meet-the-national-democratic-partyoffshoots>

11 Sur cette question, voir É. Gobe, «L’impossible politique d’épuration dans la Tunisie post-Ben Ali », in A. Allal et V. Geisser (dir.), Tunisie. Une démocratisation au-dessus de tout soupçon ?, Paris, CNRS Éditions, 2018, p. 155172.

12 Voir C. Khémira, « La recomposition du mouvement destourien », in H. Redissi, A. Nouira et A. Zghal (dir.), La transition démocratique en Tunisie : État des lieux. Les acteurs, Diwen Éditions, 2012, p. 35-69 ; J. Heurtaux, « La réinsertion des cadres de l'ancien régime en Tunisie postrévolutionnaire », Moyen-Orient, n 44, 2019, p. 26 -31. 
2011 -, et qui rassemblait d'anciens cadres du RCD avec d'anciens opposants - socialistes ou libéraux - au régime de Ben Ali. Ce parti était le fruit d'une commune opposition aux islamistes d'Ennahdha. Il devint en 2014 le plus important parti de l’Assemblée des représentants du peuple, avec 86 députés (sur 217). Dans la foulée, Beji Caïd Essebsi fut élu président de la République, avec 39,46 \% des voix au premier tour, et $55,68 \%$ au second. Avec 1,27 \% des voix, Kamel Morjane se classa alors seulement au sixième rang.

Quant à Abderrahim Zouari - ancien secrétaire général du RCD lors de la période d'ouverture relative des premières années du règne de Ben Ali - il n'obtint que 0,08 \% des voix. Son parti, le Mouvement destourien, avait été fondé un an plus tôt par Hamed Karoui - ancien cacique du PSD, puis du RCD, et Premier ministre de Ben Ali de 1989 à 1999 - dans le but de rassembler les anciens du RCD et de lutter contre leur exclusion politique. Il n’obtint aucun député lors des élections législatives de 2014. En 2016, il changea de nom pour devenir le Parti destourien libre (PDL), dans la foulée de l'élection à sa tête de Abir Moussi - ancienne secrétaire générale adjointe chargée de la femme au RCD, et avocate de ce parti lors du procès de 2011 ayant débouché sur sa dissolution. Profitant du discrédit de Nidaa Tounes - victime de son compromis historique avec les islamistes afin de former les gouvernements de 2014 à 2019 - bientôt détruit par différentes scissions, le PDL s'imposa rapidement comme l'adversaire le plus résolu d'Ennahdha. Cette stratégie lui permit d'obtenir la troisième place lors des législatives de 2019, avec 6,63\% des voix (et 4,02 \% pour sa présidente, Abir Moussi, lors des présidentielles organisées la même année). Assumant l'héritage bourguibiste et benaliste, hostile non seulement aux islamistes mais aussi aux révolutionnaires de 2011, le PDL ne conteste néanmoins pas ouvertement le cadre démocratique, se contentant de prôner une présidentialisation du régime.

En Égypte, le projet de restauration autoritaire se cristallisa au cours du premier semestre de l'année 2012 autour de la candidature à la présidentielle du général Ahmad Shafîq, ancien ministre de l'Aviation civile de Moubarak, nommé Premier ministre en pleine révolution, le 29 janvier 2011, avant de démissionner le 3 mars de la même année. À l'issue d'une campagne sans concessions, au cours de laquelle il présenta Hosni Moubarak comme un modèle et promit de rétablir l'ordre par la force si nécessaire, cet ancien général de l'armée de l'Air se classa second avec 23,7 \% des suffrages au premier tour (23-24 mai 2012), et rassembla sur son nom 48,3\% des voix au second tour (16-17 juin 2012), face au candidat des Frères musulmans Mohamed Morsi. Malgré ces résultats spectaculaires, Ahmad Shafîq n'était pas parvenu à rassembler derrière sa candidature tous les partisans de l'ancien régime. Ainsi, l'ancien ministre des Affaires étrangères de Moubarak et ancien secrétaire général de la Ligue Arabe, Amr Moussa, se présenta avec le soutien de plusieurs partis libéraux et d'anciens réformateurs. Il se classa cinquième, avec $11,1 \%$ des voix. Si la restauration autoritaire entamée en juillet 2013 sembla consacrer la victoire des anciens du PND, les divisions refirent rapidement jour parmi eux, comme le montrent notamment les projets de candidature à la présidentielle de mars 2018 du général Sâmî 'Anân - ancien numéro deux du Conseil supérieur des forces armées - et de Ahmad Shafîq lui-même. Le premier fut arrêté suite à l'annonce de sa candidature, et le second fut dissuadé d'officialiser la sienne dès son retour des Émirats arabes unis, où il avait fuit avec sa famille au lendemain du second tour de l'élection présidentielle de 2012. C'est depuis cet exil qu'il avait annoncé en septembre 2012 la création du Mouvement national égyptien.

Les élections législatives de 2015 virent ainsi une mise en compétition des partisans de l'ancien régime : réformateurs gravitant dans l'orbite de Amr Moussa ou de Esmat al-Sadate, contre-révolutionnaires du Mouvement national égyptien d'Ahmad Shafîq, proches des réseaux de Gamal Moubarak, jeunes pro-armée (parti de l'Avenir de la patrie, PAP), etc. Ces luttes reflétaient d'ailleurs en partie la compétition se jouant au sein du régime entre différents appareils sécuritaires ${ }^{13}$. Si les indépendants dominèrent le Parlement élu en 2015, dans lequel les partis politiques furent en outre représentés de manière extrêmement fragmentée (voir infra), il pourrait en être autrement lors des élections législatives prévues pour la fin de l'année 2020. En effet, les élections à la Chambre haute organisées au cours de l'été 2020 furent dominées par une « Liste nationale unifiée » qui obtint 194 sénateurs élus (sur 200, 6 sièges revenant à des indépendants), parmi lesquels 158 sont des membres du PAP. Si cette coalition pro-Sissi rassemble des organisations de gauche, des formations libérales, ainsi que le Mouvement national d'Ahmed Shafîq, le parti du Congrès (Amr Moussa), le PRD (Esmat al-Sadate), et le parti de la Liberté, il semblerait que le PAP soit désormais appelé à en devenir le parti dominant.

Proche de l'appareil sécuritaire, ce parti a été fondé en 2014 par Mohammed Badran, un étudiant de 19 ans, et avait attiré de nombreux membres de l’Union des étudiants élus sur des listes pro-Sissi. En 2015, il n’avait

13 Mada Masr, 14 mars 2016, <http://www.madamasr.com/en/2016/03/14/feature/politics/anatomy-of-an-election/> 
obtenu que 53 députés à l'Assemblée des représentants du peuple, en faisant seulement la deuxième force politique derrière le parti des Égyptiens libres (libéral, 65 sièges). Il aurait depuis lors rallié de nombreux anciens du PND, ainsi que beaucoup de députés qui n’ont néanmoins pas officialisé ce ralliement du fait de l'interdiction de changer d'affiliation partisane en cours de mandat. Mais même dans cette position où il est en passe de devenir officiellement le nouveau parti dominant, le PAP ne parvient pas à rassembler tous les anciens du PND, puisque d'autres partis issus de cette mouvance continuent à exister au sein - et même en dehors - de la « Liste nationale unifiée ». En outre, le parti des Conservateurs, l’un des partis fondés après la révolution pour défendre l'héritage du PND, est passé à l'opposition à l'occasion du référendum de mars 2019, qui avait notamment allongé la durée du mandat présidentiel et restauré la Chambre haute du Parlement. Non seulement ce parti avait alors appelé à voter contre la réforme constitutionnelle, mais il a en outre participé à l'été 2019 à des discussions avec des partis libéraux et socialistes dans le but de former une coalition d'opposition lors des législatives de 2020. Cette tentative fut néanmoins tuée dans l'œuf par l’appareil sécuritaire.

\section{De la privatisation du clientélisme à la dispersion post-révolutionnaire}

La division entre courants réformistes et conservateurs au sein des anciens partis hégémoniques ne suffit pas à épuiser la compréhension de l'extrême dispersion de leurs membres après les révolutions de 2011. Cette dispersion a en effet été aggravée par la structuration clientélaire de ces deux formations.

Héritiers de partis uniques, le RCD et le PND s'étaient comportés dans un premier temps comme des partis centralisés aux mains de la haute administration, permettant à cette dernière de coopter à sa guise les élites locales, et peuvent de ce fait être décrits comme des partis de l'État et de l'administration ${ }^{14}$. Les deux partis connurent néanmoins au cours des années 1990 et 2000 un phénomène de "privatisation » des réseaux de clientèle. En Tunisie, ce phénomène fut l'œuvre des « clans » issus de l'entourage de Ben Ali, et rassemblés autour de la famille de sa femme, Leïla Trabelsi. Ces quelques dizaines de familles assurèrent leur domination sur la vie politique et économique tunisienne au cours des années 1990 et 2000. Le rôle du RCD dans la distribution de la protection sociale - bénéfice attendu de la négociation par les plus fragiles - avait alors fait émerger à l'échelle locale la figure des « big men multipositionnés " ${ }^{15}$, notables s'imposant comme bienfaiteurs au nom du régime et du parti, facilitant ici l'accession à l'emploi public, là l'obtention d'une licence de taxi. À cette échelle, la privatisation des réseaux de clientèle prenait la forme à la fois d'une concentration de la petite corruption décentralisée au profit des « clans » de l'entourage présidentiel - dont les Trabelsi étaient devenus le symbole - et d'une captation des bénéfices par ces mêmes « clans » en lieu et place du régime et du RCD. Dès la fin de la décennie 1990, les proches du régime et de son président ne se limitaient plus à la prédation sur les grands contrats et les investissements étrangers ${ }^{16}$ mais tendaient à s'imposer comme intermédiaires sur une gamme de plus en plus large d'opérations économiques, jusqu'à celles de l'économie informelle qui se mondialisait alors à mesure que des sources de biens de consommation bon marché émergeaient, en particulier en Chine, et que les acteurs marchands cherchaient à contourner avantageusement les barrières douanière du pays ${ }^{17}$.

Le dispositif clientéliste que les membres des «clans » avaient mis sur pied articulait le trafic de leur influence politique et économique et le recel de leur impunité acquise dans la décennie 1990 tant sur les marchés publics tunisiens que sur les investissements privés dans le pays. Le système qui fut progressivement mis en place était assez simple. Il s'agissait pour les hommes d'affaires proches du pouvoir de proposer aux commerçants un accès à l'importation transnationale libre de contrôles et de droits de douane. En échange d'une telle garantie, ces clients s'acquittaient d'une rétribution auprès de leurs bienfaiteurs. Ce dispositif reposait sur trois éléments-clés : la mise au pas des contrôles de l’État, à commencer par les services des douanes portuaires, en mêlant intéressements et pressions ; la création de sociétés commerciales et/ou logistiques intouchables et au nom desquelles étaient réalisées les importations

14 Sur la notion de parti de l'administration, voir par exemple L. Addi, " Les partis politiques en Algérie », Revue des Mondes musulmans et de la Méditerranée, n 111-112, 2006, p. 139-162.

15 A. Allal, « Retour vers le futur. Les origines économiques de la révolution tunisienne », Pouvoirs, n 156, 2016, p. 17-29.

16 C. Freund, A. Nucifora, B. Rijkers, All in the Family. State Capture in Tunisia, The World Bank, Policy Research Working Paper 6810, 2014.

17 A. Doron, «Devenir importateur transnational en Tunisie : articulations entre mobilités et relations sociales », Espace, populations, sociétés, $\mathrm{n}^{\circ} 2,2017<\underline{\text { https://journals.openedition.org/eps/7170\#tocto2n3 }>}$ 
du secteur informel ; un démarchage actif de petits commerçants candidats à l'importation. Les artisans de la mise en œuvre de ce dispositif n'étaient toutefois pas directement les membres de la famille présidentielle élargie mais les transitaires, de puissants intermédiaires agissant en leur nom. C'est sur ce dispositif qu'a reposé l'expansion du commerce informel de biens importés dans la décennie 2000, en particulier à Tunis avec le développement des souks de Boumendil et Moncef Bey ${ }^{18}$. Cette domination des "clans » priva progressivement le gros de la base du RCD d'accès aux ressources du parti, et réduisit donc leur capacité à entretenir leurs propres clientèles ${ }^{19}$.

En Égypte, la privatisation du clientélisme fut au contraire une conséquence de l'élargissement de la base sociale du PND, et plus précisément de la cooptation de nouvelles élites de businessmen issus des réformes économiques, et qui venaient concurrencer les élites traditionnelles. Ainsi, à partir de 1990, une compétition de plus en plus aiguë se mit en place au niveau local, sans remettre en cause le cadre offert par le PND. Ce dernier se transforma ainsi progressivement en structure lâche, une sorte d'arène au sein de laquelle les potentats locaux s'affrontaient pour accéder aux ressources du pouvoir central en mobilisant dans la lutte leurs ressources propres. Ainsi, dans les deux pays, et pour des raisons différentes, les notables devaient de plus en plus compter sur leurs propres ressources. Lorsque le marché politique fut libéralisé, en 2011, une grande partie d'entre eux s'éparpilla, entre candidatures indépendantes et ralliement à différents partis situés sur l'ensemble du spectre politique. Cette situation explique pourquoi la dissolution du RCD et du PND a provoqué dans un premier temps un sauve-qui-peut généralisé parmi leurs anciens membres, alors qualifiés en Égypte du sobriquet de feloul, terme désignant les « résidus d’une armée en déroute ».

La révolution de 2011 a constitué une soudaine poussée de politisation, modifiant en profondeur le paysage politique : alors que celui-ci était jusqu'alors structuré par une échelle de proximité ou d'éloignement par rapport au cœur du pouvoir, il s'est trouvé brusquement polarisé autour de profonds clivages sociaux ${ }^{20}$. Dans ce contexte, beaucoup d'anciens membres du RCD et du PND se sont ré-alignés, en rejoignant des partis correspondant à la défense des intérêts de leurs différents groupes sociaux. Cette politisation d'une partie des élites anciennement satellisées par les partis au pouvoir a été favorisée par un système électoral faisant la part belle à la proportionnelle ${ }^{21}$. Ainsi, le recyclage des feloul n'a pas uniquement concerné les petits partis se disputant l'héritage des anciens partis hégémoniques (voir supra) mais la quasi-totalité du spectre politique. Dans les deux pays, on constate par ailleurs qu'à partir de 2012, une commune opposition aux islamistes a permis aux ex-RCDistes et ex-PND de se rapprocher des libéraux, des socialistes et des révolutionnaires de 2011, et de récupérer ainsi une certaine légitimité.

En Égypte, les élections de 2011-2012 ont vu les feloul se présenter sous les bannières de quasiment tous les partis et coalitions en lice ${ }^{22}$. Lors des élections de 2015 également, d'anciens membres du PND ont porté les couleurs de partis politiques aux positionnements différents, voire opposés. Avec 325 députés sur 568 élus, les indépendants dominèrent cependant cette assemblée. La représentation partisane y fut en outre extrêmement fragmentée (le parti arrivé en tête, celui des Égyptiens libres, occupait seulement 65 sièges). Le retour en force du scrutin majoritaire, et la réduction subséquente de la taille des circonscriptions avait bien entendu favorisé ce retour des indépendants, mais il convient de noter que ces derniers avaient obtenu proportionnellement davantage d'élus au scrutin de liste (74 sur 120, soit 61,7 \%) qu'au scrutin majoritaire (251 sur 448, soit 56 \%). Ceci s'explique par la composition de la liste arrivée en tête dans les quatre circonscriptions. Baptisée "Pour l'amour de l'Égypte », celle-ci visait à réaliser l'union nationale en rassemblant plusieurs partis (des Égyptiens libres au PAP), mais comportait également une majorité de candidats indépendants sélectionnés par les organisateurs de la liste sur des critères de popularité et de

18 A. Doron, Routes tunisiennes de l'échange marchand. Géographie post-révolution d'un réseau de marchés mondialisés, Toulouse, Université Toulouse 2 Jean-Jaurès, thèse de doctorat en géographie, 2018.

19 A. Wolf, op. cit., p. 253-254.

20 C. Steuer, « Recomposition du champ politique et enjeux électoraux », in B. Rougier et S. Lacroix (dir.), L’Égypte en révolutions, Paris, Presses universitaires de France, 2015, p. 99-122.

21 C. Steuer, «Le printemps des partis ? Le rôle des organisations partisanes égyptiennes dans les élections législatives », Confluences Méditerranée, $\mathrm{n}^{\circ}$ 82, 2012, p. 91-105. Pour une étude de cas, voir G. du Roy, " La campagne d'Al-Misriyyîn Al-Ahrâr chez les chiffonniers de Manchiyit Nâsir », Égypte/Monde arabe, $3^{\mathrm{e}}$ série, $\mathrm{n}^{\circ} 10$, 2013.

22 À l'exception de la coalition de « La Révolution continue ». C. Steuer, "Representing the people in the street or in the ballot box? The revolutionary coalition campaign during the 2011 Egyptian elections », Mediterranean Politics, $2019<$ https://www.tandfonline.com/doi/full/10.1080/13629395.2019.1673572> 
réputation. Dans l'ensemble, les anciens membres du PND furent représentés dans cette assemblée ${ }^{23}$, mais moins bien que ce à quoi ils s'attendaient ${ }^{24}$. Par ailleurs, ces élections virent se développer massivement un phénomène difficile à chiffrer avec précision : l'entrée au Parlement de jeunes parents des anciens élus PND, se lançant en politique afin de conserver dans la famille un siège de députée ${ }^{25}$. En Tunisie, une partie des anciens du RCD est revenue aux affaires à la faveur de la victoire de Nidaa Tounes et de Beji Caïd Essebsi en 2014. En mai 2019, l’Initiative destourienne annonça sa fusion avec Tahya Tounes, parti du Premier ministre Youssef Chahed, issu d'une scission de Nidaa Tounes. En août de la même année, Kamel Morjane devint même Premier ministre par délégation lorsque Youssef Chahed entra en campagne pour les présidentielles. À l'automne 2020, la question du rôle politique des élites issues du RCD était au cœur de la crise opposant le président de la République Kaïs Saïd à son Premier ministre Hicham Mechichi ${ }^{26}$.

La période de transition fut aussi celle d'une active recomposition des imbrications politico-économiques sur lesquelles reposaient les importations informelles. En effet, si Ben Ali, les « clans » et le RCD ont disparu, ce n'est guère le cas de l'ensemble des cadres de l'ancien parti présidentiel, des intermédiaires affiliés au régime ou aux « clans », des « big men » et autres notables locaux qui entretenaient des réseaux d'allégeance et/ou d'affaires, comme bienfaiteurs ou pour le compte des anciens " clans » prédateurs. De même que la dissolution du RCD a laissé indemne nombre de ces "entremetteurs sociaux » à même d'exploiter leurs réseaux de relations dans un contexte d'incertitudes ${ }^{27}$, cette analyse de la redistribution politique des intermédiaires de l'ancien régime est tout à fait valable pour la sphère économique, tant leur savoir-faire s'était constitué à la charnière des deux mondes. Ces entrepreneurs en négociation aux profils variés anciens notables du RCD, agents gradés de l'État (douanes), hommes d'affaires, anciens transitaires reprenant à leur compte les réseaux qu'ils opéraient jusqu'en 2011 - participèrent ainsi d'une recomposition des réseaux économiques du temps de Ben Ali. Recomposition, et non rétablissement, car ces acteurs ne sont plus au service d'un système économico-politique centralisé, mais agissent désormais dans un paysage dérégulé où la concurrence est grande et où des nouveaux venus s’insèrent à toutes les échelles.

En Égypte, le phénomène des " entrepreneurs militaires $»^{28}$, qui avait commencé à se développer dans les années 1990, prend depuis le renversement de Muhammad Morsi une ampleur jamais égalée dans le passé. Ainsi, les réseaux de clientèle se sont réorganisés autour de l'armée, en tant qu'acteur économique de premier plan. Cette réorganisation a été accélérée par la politique de grands travaux initiée par le régime (extension des capacités du canal de Suez, construction d'une « nouvelle capitale administrative »). Du fait de leurs avantages comparatifs (exemption d'impôts, recours aux conscrits comme main-d'œuvre bon marché et non syndiquée, accès privilégié à l'import et à l'export), les entrepreneurs militaires sont en position d'évincer les entrepreneurs civils qui refuseraient de rentrer dans un rapport de clientèle avec eux. En septembre 2019, les déclarations publiques de Muhammad Ali, un ancien contractant de l'armée autoexilé en Espagne, ont levé le voile sur une partie de ces pratiques ${ }^{29}$.

En 2011, la chute du régime de Ben Ali avait dans un premier temps stoppé net les importations du secteur informel via les ports tunisiens. L’activité s'était alors repliée pour certains et redéployée pour d'autres, encourageant les importations contournant les barrières douanières par les frontières terrestres du pays. Cependant, tous les commerçants qui avaient pratiqué l'importation transnationale et les voyages d'affaires en Chine ${ }^{30}$ cherchaient activement de nouveaux intermédiaires et de nouveaux protecteurs. Un des effets

23 Al-Masry al-youm, 29 octobre 2015, <http://www.almasryalyoum.com/news/details/835098>

24 M. Abou Kassem, et. al., « Dépolitisation de l'urne et politisation de l'électeur en Égypte : quelques enseignements des élections législatives égyptiennes 2015 », Confluences Méditerranée, n 102, 2017, pp. 181-196.

25 Ibid. Voir aussi : A. A. H. Hussien, « Egypt’s 2015 Parlementarians, New Faces - Old Logic », Policy Alternatives, 2016, <http://www.arab-reform.net/en/node/918> ; W. 'Atalm, " Al-Jîl al-thânî min al- 'â'ilât wa- 'awda shabaka almasâlih al-taqlîdiyya (La seconde génération des grandes familles et le retour des réseaux d’intérêts traditionnels) », Rû́â misriyya (Vision égyptienne), n 11, 2015, p. 24-28.

26 Al-'Arab, 25 septembre 2020, < $\underline{\text { https://frama.link/Su41AzFW> }}$

27 A. Allal, V. Geisser, « La Tunisie de l'après-Ben Ali », Cultures \& Conflits, $\mathrm{n}^{\circ}$ 83, 2011 $<$ https://journals.openedition.org/conflits/18216>

28 Z. Abul-Magd, "Egypt’s Adaptable Officers. Business, Nationalism, and Discontent », in Z. Abul-Magd and E. Grawert (eds), Businessmen in Arms. How the Military and Other Armed Groups Profit in the MENA Region, London, Rowman \& Littlefield, 2016, pp. 23-41.

29 Middle East Monitor, 4 septembre $2020<$ https://www.middleeastmonitor.com/20190904-egypt-contractor-accusesarmy-of-squandering-public-funds-amid-austerity/>

30 S. Belguidoum, O. Pliez, "Made in China. Commerce transnational et espaces urbains autour de la Méditerranée », Les Cahiers d'EMAM, n² 26, 2015 < https://journals.openedition.org/emam/909> 
perceptibles de telles recompositions post-révolutionnaires a été la reprise progressive des importations de l'économie informelle, et leur réorientation via des ports secondaires qui ont vu leur trafic exploser (Sfax, Bizerte, Sousse $)^{31}$. En témoignent quelques affaires, dont celle de Sfax en $2015^{32}$, à l'occasion de laquelle a été mis à jour un réseau de contrebande de feux d'artifices impliquant des hommes d'affaires de la région, des gradés des douanes et des commerçants, répliquant, après la chute du régime et à une échelle plus modeste, le dispositif de contournement opéré par les « clans ». Comme le décrivaient les acteurs marchands du secteur informel quelques années après la révolution, « au lieu d'avoir dix Trabelsi, maintenant, tu en as cent $»^{33}$, et cette tendance semble s'être accentuée au même rythme que les difficultés économiques et politiques post-révolution.

Ce phénomène peu évoqué dans les médias et le débat public est pourtant reconnu par des cadres de la Direction Générale des Douanes, du patronat, des ministères, de la plupart des partis politiques, et même par d'anciens intéressés tel qu'Imed Trabelsi admettant en 2017 que "le même système est toujours opérationnel $\aleph^{34}$. Pourtant, les imbrications politiques et économiques des importations de l'économie informelle en Tunisie sont peu traitées, au profit d'une approche dénonçant la contrebande des régions frontalières et agitant l'épouvantail de collusions avec le terrorisme. Or, cela détourne l'attention de l'opinion publique et des gouvernants de l'organisation réelle du secteur informel marchand et de cet échec majeur de la révolution. L'organisation clientéliste de l'économie informelle a non seulement survécu à la révolution et à la disparition des têtes de réseau, mais elle a été reproduite, étendue, généralisée. Cette contre-révolution économique répliquant les prédations semble aujourd'hui l'emporter. Sa généralisation est devenue à ce point crédible qu'on lui attribue désormais dans les ministères la hausse des prix des fruits et légumes ou celle du tabac, censés être régulés par l’État. Les principales formations politiques s'accusent d'ailleurs mutuellement de complaisance voire d'implication dans ces réseaux et ce jusqu'aux fonctions gouvernementales, suspicion généralisée reconnue par des cadres des Douanes ou par l'INLUCC ${ }^{35}$, qui conviennent d'ailleurs que les partis politiques - comme le port de Radès qui centralise le trafic de conteneurs - sont intouchables.

\section{Conclusion}

Malgré des différences évidentes dans le fonctionnement de leurs systèmes politiques respectifs, la Tunisie et l'Égypte ont toutes deux vu le retour aux affaires d'une partie du personnel de l'ancien régime, résultat tant de stratégies de reconversions individuelles que d'entreprises de recyclages collectifs. Ces différents projets ont débouché sur divers degrés d’intégration aux nouveaux régimes. L’héritage de l'ancien régime n’est jamais totalement assumé, même si ce dernier est rarement rejeté en bloc. Les entreprises de restauration les plus franches préfèrent se référer au bourguibisme et au destourisme en Tunisie, et au " nasser-sadatisme " en Égypte ${ }^{36}$, tout en se bornant à condamner certains " excès » des régimes de Ben Ali et de Moubarak. De fait, la cohérence et les objectifs du projet contre-révolutionnaire sont à rechercher pour l'essentiel en dehors du champ politique. En effet, l'observation de la crise de la fonction clientéliste du RCD du point de vue de l'économie informelle permet d'éclairer « par le bas » à la fois l'inaptitude du parti hégémonique à survivre à la chute du dictateur, et le succès d'un modèle économique forgé sous l'ancien régime. Ainsi l'héritage des

31 A. Doron, op. cit., p. 2-5.

32 Le Temps, 4 avril $2015<$ http://www.letemps.com.tn/article/90590/le-groscoup-de-filet-de-sfax-mettra-t-il-fin-au-ph \%C3\%A9nom\%C3\%A8ne>

33 Entretien, souk Boumendil, Tunis, printemps 2015.

34 Le Monde, 20 mai 2017 <http://www.lemonde.fr/afrique/article/2017/05/20/a-tunis-un-membre-du-clan-ben-alirevele-lampleur-de-la-corruption-de-l-ancien-regime 5130974 3212.html $>$

35 Instance Nationale de Lutte Contre la Corruption.

Entretiens, Tunis, octobre 2019 et février 2020.

Cette suspicion transcende l'ensemble du spectre partisan post-révolution. Le parti islamiste Ennahda, par exemple, est davantage cité en lien avec les réseaux de la contrebande frontalière, notamment par ses ancrages dans la région frontalière sud-est. Cependant, ce lien évoqué ne renvoie pas aux nécessairement aux anciens dispositifs de contournement portuaires des « clans ». Si les mécanismes clientélistes sont identifiables, leur opacité brouille les pistes et doit inciter à la prudence avant d'incriminer une formation politique, car ces accusations sont aussi des outils de décrédibilisation dont les adversaires politiques de tout bord ne se privent pas.

36 Orient XXI, $2016<$ https://orientxxi.info/magazine/le-nasser-sadatisme-un-phenomene-politique-et-culturel-dans-legypte-de-sissi,1153> 
mécanismes clientélistes du RCD, privatisés par les «clans» prédateurs proches du pouvoir, s'est autonomisé de l'ancienne structure partisane comme des anciennes hiérarchies du régime. Et c'est ce découplage observé depuis l'économie informelle qui fait figure de projet contre-révolutionnaire diffus sans nom, ni porteur, ni réel opposant. 